\title{
Tooth Contour Extraction for Matching Dental Radiographs
}

\author{
Hong Chen and Anil K. Jain \\ Department of Computer Science and Engineering \\ Michigan State University \\ East Lansing, MI, 48823 \\ Email: \{chenhon2,jain\}@cse.msu.edu
}

\begin{abstract}
In dental biometrics [1] [2], the contours of teeth in dental radiographs are utilized for human identification. However, in many images, the contours of teeth are fuzzy and only partially visible. To extract the contours from these images, we propose a method based on the active contour models [4]. A new dynamic energy is proposed for the directional snake to discriminate boundaries of adjacent teeth. Matching result shows the contours extracted with this method perform better than traditional methods.
\end{abstract}

\section{Introduction}

Dental biometrics automatically analyzes dental radiographs to identify the deceased individuals. The radiographs acquired after a person is deceased are called the Post-mortem (PM) radiographs, and the radiographs acquired while the person is alive are called the Ante-mortem (AM) radiographs (Figure 1). The AM radiographs, collected from the dentists, are labelled with the patient names. The approach for human identification is to match the PM radiographs of the unidentified person against the database of AM radiographs. If the set of teeth in the PM images matches the teeth in an AM radiograph, then the identity of that person is obtained.

The contours of the teeth are important features in dental biometrics. They can be directly utilized as cues for identification [1] [2]. To extract the contours, Jain et al. [1] proposed a method based on edge detection, and Jain and Chen [2] proposed an extraction method by classifying the pixels in the radiographs using an intensity distribution model. However, these methods are subject to big errors when the tooth contours are fuzzy and only partially visible.

In this paper, we propose a contour extraction method based on the active contour models [4]. To discriminate the

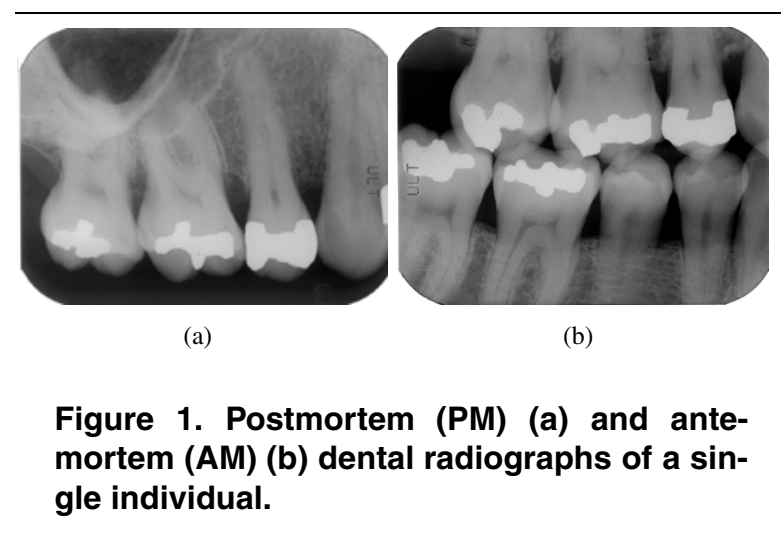

boundaries of neighboring teeth, we propose a new dynamic energy term for the directional snake.

\section{Directional Snake}

The traditional snake is not able to discriminate edges of multiple adjacent objects. As a result, the snake may partly overlap on the boundary of the neighbored objects. A solution to this problem is to utilize the gradient directions if all the boundaries of objects have consistent 'inward' or 'outward' gradient. Dental radiographs satisfy this requirement, because the teeth have higher intensities than their surroundings, so the gradient directions of the edges are consistently toward the exterior of the teeth. If the snaxles are in clockwise order, then the gradient at each snaxle should always point to the left of the snake.

Park et al. [5] proposed the directional snake to utilize the direction information. Their method is to redefine the dynamic external energy in the following way:

$$
E_{\text {ext }}\left(s_{i}\right)= \begin{cases}-\left\|G\left(s_{i}\right)\right\|, & \text { if } \theta_{G}\left(s_{i}\right)-\theta_{N}\left(s_{i}\right) \leq \frac{\pi}{2} \\ 0, & \text { otherwise, }\end{cases}
$$

where $\left\|G\left(s_{i}\right)\right\|$ is the gradient magnitude at the $i$ th snaxel 
$s_{i}, \theta_{G}\left(s_{i}\right)$ is the gradient direction at $s_{i}$, and $\theta_{N}\left(s_{i}\right)$ is the contour's normal direction at $s_{i} . \theta_{N}\left(s_{i}\right)$ is defined as

$$
\theta_{N}\left(s_{i}\right)=\theta_{C}\left(s_{i}\right)+\lambda_{i} \frac{\pi}{2},
$$

where $\theta_{C}\left(s_{i}\right)$ is the counterclockwise tangent direction at snaxel $s_{i}$, and $\lambda_{i}= \pm 1$ controls the angle between the normal direction and the tangent direction $(\pi / 2$ or $-\pi / 2)$, so that snaxel $s_{i}$ converges to rightward gradient or leftward gradient. The shortcoming of this method is that it sets the external energy to zero when the snaxels are in the energy field of those boundaries whose gradient directions are opposite to what we want. It causes the snaxels to lose the guidance of the external force.

We define a new external energy at $s_{i}$ as:

$$
E_{\text {ext }}\left(s_{i}\right)=-\left\|G\left(s_{i}\right)\right\| \cos \left(\theta_{G}\left(s_{i}\right)-\theta_{N}\left(s_{i}\right)\right) .
$$

The variation of $E_{\text {ext }}\left(s_{i}\right)$ with $\left\{\theta_{G}\left(s_{i}\right)-\theta_{N}\left(s_{i}\right)\right\}$ is shown in Figure 2.

This definition shows that when $\theta_{G}\left(s_{i}\right)$ is in the direction of $\theta_{N}\left(s_{i}\right)$, i.e., $\left|\theta_{G}\left(s_{i}\right)-\theta_{N}\left(s_{i}\right)\right|<\pi / 2$, the external energy draws the snaxel near; When $\theta_{G}\left(s_{i}\right)$ is in the opposite direction of $\theta_{N}\left(s_{i}\right)$, i.e., $\left|\theta_{G}\left(s_{i}\right)-\theta_{N}\left(s_{i}\right)\right|>\pi / 2$, the external energy pushes the snaxel away. By comparison, this definition is superior to Equation (1) in that even when the gradient direction is not favorable to the contour direction, the snake can still interact with the inverted external energy, which actually pushes the snake away from that edge.

Figure 3 shows how the external energy field inverts as the direction of the snake changes.

\section{Contour Extraction Process}

The process of the tooth contour extraction involves three stages: initialization, convergence to the gradient, and fine adjustment. The details of each stage are given in the following subsections.

\subsection{Initialization}

Jain and Chen [2] proposed the radiograph segmentation method, which uses the integral projection to detect the gap between the upper and lower teeth and the gap between neighboring teeth (see Figure 4(a)). The segmentation result forms the region of interest (ROI) for each tooth.

The gumline is used to separate the crowns and the roots of the teeth for the snake initialization. The gum line detection method utilizes the property that there is an intensity increasement at the gum lines from the crown area to the root area. So, a horizontal snake is initialized in the middle

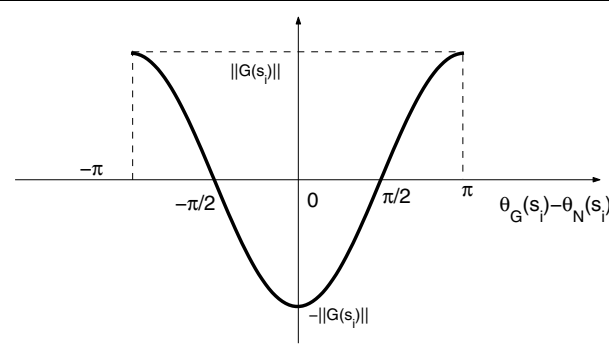

Figure 2. The magnitude of the external energy changes with the angle of $\left\{\theta_{G}\left(s_{i}\right)-\right.$ $\left.\theta_{N}\left(s_{i}\right)\right\}$.

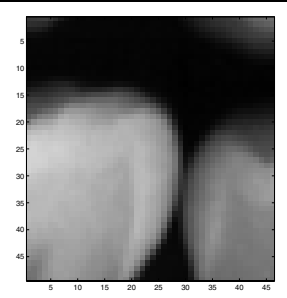

(a)

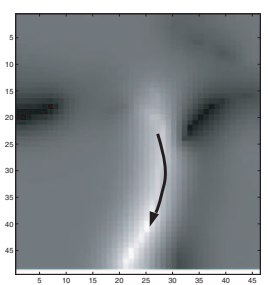

(b)

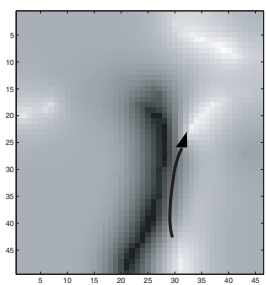

(c)
Figure 3. The magnitude of the energy field inverts as the direction of the snake changes. The snakes are designed to converge to the boundary where the gradient is perpendicular and the interior intensities are higher than exterior intensities. (a) When the snake is downward, the boundary of the left tooth attracts it, while the boundary of the right tooth repels it. (b) When the snake is upward, the boundary of the right tooth attracts it, while the boundary of the left tooth repels it. 


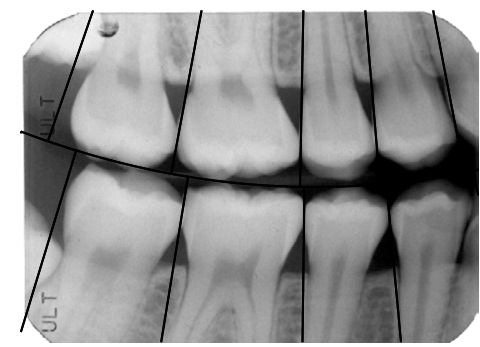

(a)

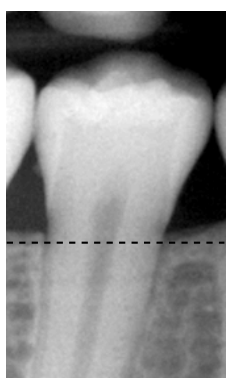

(b)

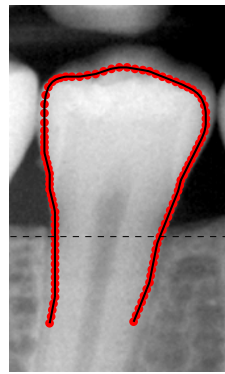

(d)

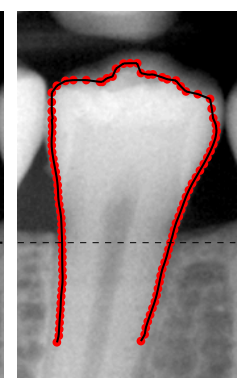

(c)

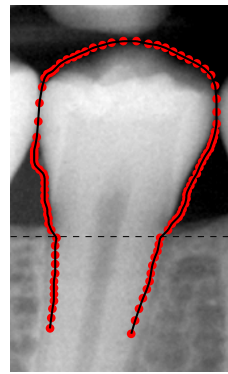

(e)
Figure 4. Stages in the tooth contour extraction: (a) radiograph segmentation; (b) gum line detection; (c) initialization; (d) convergence to the gradient; (e) fine adjustment of the crown contour.

of the ROI, and it finally converges to the state that the average of the vertical gradient components along the snake is maximized (see Figure 4(b)).

The snake of the tooth shape is initialized with the pixel classification method proposed by Jain and Chen [2] (see Figure 4(c)).

\subsection{Convergence to Gradient}

The Gradient Vector Flow (GVF) field of the edges detected by the Canny operator is used as the external energy field [6]. A GVF field of a tooth image is shown as an example in Figure 5.

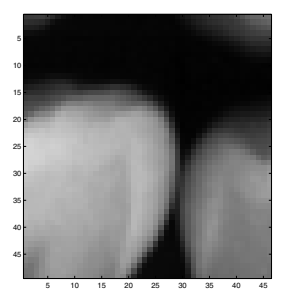

(a)

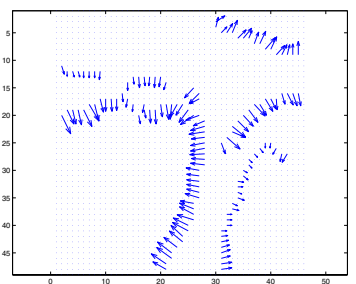

(b)

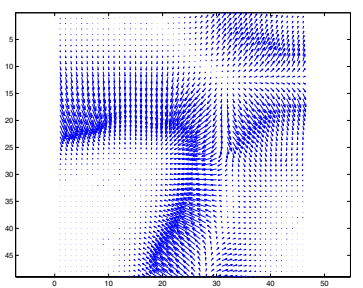

(c)
Figure 5. An example of GVF field of a tooth image: (a) the intensity image; (b) the gradient of the intensity image; (c) the GVF field of the gradient image.

Define the dynamic external energy as Equation (3) with $\lambda_{i}=1$. So, $s$ converges to the boundaries, where the gradient is perpendicular to $s$ and the intensities of the interior pixels of $s$ are larger than those of the exterior pixels (Figure $4(d))$.

\subsection{Fine Adjustment}

Due to the 3D structure of the teeth, the largest gradient usually does not take place exactly at the true boundaries of teeth. So the result of the previous stage needs to be adjusted to converge to the true boundaries. We utilize the property that the true boundaries usually touch with area of lower intensities. So, the external energy is defined as

$$
E_{\text {ext }}=E_{\text {ext }, 1}+\omega E_{\text {ext }, 2},
$$

where $E_{e x t, 1}$ is defined as equation (3), $E_{\text {ext,2 }}$ is the intensity image, and $\omega$ controls the trade-off between the gradient and the intensity. The result of this step is shown in Figure 4(e) and Figure 6.

\section{Experimental Results}

The tooth contours extracted from AM and PM images are matched for retrieval. The matching distances are the 


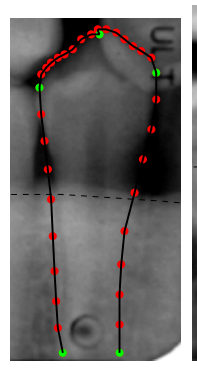

(a)

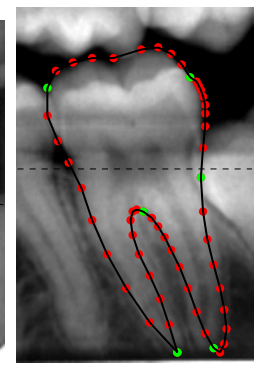

(b)
Figure 6. Some results of the contour extraction.

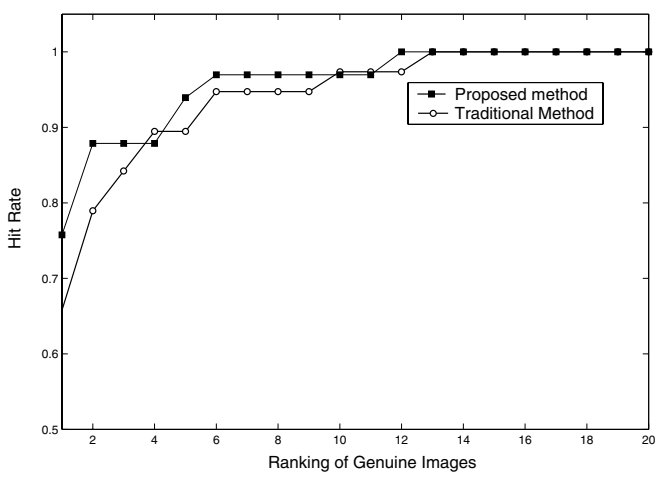

(a)

Figure 7. Matching results with the contours extracted with the proposed method and with the method proposed in [1].

similarity measurement of the teeth from the two images.

The matching distance between two contours, $A$ and $B$, is defined as [1]:

$$
M D(A, B)=\min _{T} \frac{1}{|A|} \sum_{a \in A}\left(\min _{b \in B}\|a, T(b)\|\right),
$$

where $\|$.$\| is the Euclidean distance between two points on$ the two curves, and $T$ is the rigid transformation which aligns two curves to minimize the distance. The optimization procedure uses the sequential quadratic programming (SQP) method [3].

The result of retrieving 38 images from a database of 130 images is shown in Fig. 7. For most ranks of genuine images, the contours extracted with this new method perform better than those extracted with the method presented in [1].

\section{Conclusion and Discussion}

A systematic tooth contour extraction method is proposed in this paper. There are three stages: initialization, convergence to gradients and fine adjustment. The initialization includes radiograph segmentation, gum line detection, and initialization of the snakes. The gradient vector flow (GVF) field is used as the external energy field, and an improvement in the dynamic energy term of the directional snake is proposed for discriminating neighboring teeth. After the snake converges to the gradient, a composite energy field is used to finely adjust the snake so that the true boundaries are detected. Matching results show the contours extracted with this method perform better than the traditional methods [1] [2].

Due to the limited discriminability offered by the tooth contours, we are exploring the use of dental work (e.g., fillings, crown) for matching.

\section{Acknowledgment}

This research was supported by the National Science Foundation grant EIA-0131079.

\section{References}

[1] A. K. Jain, H. Chen, and S. Minut. Dental biometrics: Human identification using dental radiographs. In Proc. of 4th AVBPA, Guildford, UK, pages 429-437, June, 2003.

[2] A. K. Jain and H. Chen. Matching of dental X-ray images for human identification. Technical Report MSU-CSE-03-33, Department of Computer Science, Michigan State University, East Lansing, Michigan, November 2003.

[3] T. F. Coleman and Y. Li. An interior, trust region approach for nonlinear minimization subject to bounds. SIAM Journal on Optimization, Vol. 6:pp. 418-445, 1996.

[4] M. Kass, A. Witkin, and D. Terzopoulos. Snakes: Active contour models. Int. J. Comput. Vis., 1:321-331, 1987.

[5] H. Park, T. Schoepflin, and Y. Kim. Active contour model with gradient directional information: Directional snake. IEEE Transactions on Circuits and Systems for Video Technology, 11(2):252-256, 2001.

[6] C. Xu and J. L. Prince. Snakes, shapes, and gradient vector flow. IEEE Transactions on Image Processing, 7(3):359-369, March 1998. 